\title{
THE MEDIAN PROBLEM ON $k$-PARTITE GRAPHS
}

\author{
KARUVACHERY PRAVAS \\ Government Polytechnic College \\ Koratty-680 308 \\ India \\ e-mail: pravask@gmail.com \\ AND \\ AMBAT ViJAYAKUMAR \\ Cochin University of Science and Technology \\ Cochin-682022 \\ India \\ e-mail: vambat@gmail.com
}

\begin{abstract}
In a connected graph $G$, the status of a vertex is the sum of the distances of that vertex to each of the other vertices in $G$. The subgraph induced by the vertices of minimum (maximum) status in $G$ is called the median (anti-median) of $G$. The median problem of graphs is closely related to the optimization problems involving the placement of network servers, the core of the entire networks. Bipartite graphs play a significant role in designing very large interconnection networks. In this paper, we answer a problem on the structure of medians of bipartite graphs by showing that any bipartite graph is the median (or anti-median) of another bipartite graph. Also, with a different construction, we show that the similar results hold for $k$-partite graphs, $k \geq 3$. In addition, we provide constructions to embed another graph as center in both bipartite and $k$-partite cases. Since any graph is a $k$-partite graph, for some $k$, these constructions can be applied in general.
\end{abstract}

Keywords: networks, distance, median, bipartite, $k$-partite.

2010 Mathematics Subject Classification: 05C12.

\section{REFERENCES}


[1] K. Balakrishnan, B. Brešar, M. Kovše, M. Changat, A.R. Subhamathi and S. Klavžar, Simultaneous embeddings of graphs as median and antimedian subgraphs, Networks 56 (2010) 90-94.

doi:10.002/net.20350

[2] R. Balakrishnan and K. Ranganathan, A Textbook of Graph Theory, Second Edition (Heidelberg, Springer, 2012).

doi:10.1007/978-1-4614-4529-6

[3] H. Bielak and M.M. Sysło, Peripheral vertices in graphs, Studia Sci. Math. Hungar. 18 (1983) 269-275.

[4] H. Kautz, B. Selman and M. Shah, Referral Web: combining social networks and collaborative filtering, Communications of the ACM 40(3) (1997) 6365.

doi:10.1145/245108.245123

[5] K. Pravas and A. Vijayakumar, Convex median and anti-median at prescribed distance, communicated.

[6] P.J. Slater, Medians of arbitrary graphs, J. Graph Theory 4 (1980) 389-392. doi:10.1002/jgt.3190040408

[7] S.B. Rao and A.Vijayakumar, On the median and the anti-median of a cograph, Int. J. Pure Appl. Math. 46 (2008) 703-710.

[8] H.G. Yeh and G.J. Chang, Centers and medians of distance-hereditary graphs, Discrete Math. 265 (2003) 297-310.

doi:10.1016/S0012-365X(02)00630-1

Received 24 February 2014

Revised 20 June 2014

Accepted 15 August 2014 